\title{
Reversing Bonferroni
}

\author{
Gregory Francis ${ }^{1}$ Evelina Thunell ${ }^{1,2}$
}

Accepted: 22 November 2020 / Published online: 19 January 2021

(C) The Psychonomic Society, Inc. 2021

\begin{abstract}
It is common for conclusions of empirical studies to depend on multiple significant outcomes. This practice may seem reasonable, but it has some unintended effects. In particular, the compound Type I error rate for multiple studies (the likelihood of concluding that an effect exists when it does not) can be much lower than that of the individual studies. This in itself is not a problem since a low Type I error rate is desirable. However, there is also an accompanying drop in power, meaning that the probability of finding support for a true effect is low. Currently, there is no standard statistical method for dealing with the hyperconservative error rate and accompanying low power that results from investigations requiring multiple significant outcomes. Here, we propose a novel solution to this problem: We show that it is sometimes appropriate to reverse the logic of the classic Bonferroni correction and increase the significance criterion in order to maintain an intended compound Type I error rate across multiple tests. This reverse Bonferroni approach dramatically improves statistical power and encourages careful planning of statistical analyses prior to data collection. To avoid adding to the list of questionable research practices that seem to contaminate some psychological research, we suggest that reverse Bonferroni be restricted to situations where authors pre-register their analysis plans.
\end{abstract}

Keywords Bonferroni $\cdot$ Error rate $\cdot$ Power $\cdot$ Replication

\section{Introduction}

The standard approach to null hypothesis testing defines a decision-making procedure that produces a Type I error (concluding that an effect exists when it does not) at a set rate, typically 0.05 . Due to concerns about misuse of hypothesis testing and questionable research practices (John, Loewenstein, \& Prelec, 2012; Simmons, Nelson, \& Simonsohn, 2011) many scientists will not conclude support for an effect without first running independent replications to verify the key findings (Cohen, 1994; Nosek, Spies, \& Motyl, 2012; Roediger, 2012). While this cautious approach might seem reasonable, it is important to realize that requiring multiple test outcomes entails a non-intended decrease in the Type I error rate, which further leads to an increased Type II error

Gregory Francis

gfrancis@purdue.edu

1 Department of Psychological Sciences, Purdue University, 703 Third Street, West Lafayette, IN 47907-2004, USA

2 Department of Clinical Neuroscience, Karolinska Institutet, Nobels väg 9, 17177 Stockholm, Sweden (not concluding an effect exists when it actually does exist) rate. Throughout this manuscript, we refer to the Type I error rate for a decision that is based on multiple test outcomes as the compound Type I error rate.

When conducting multiple tests, scientists often use Bonferroni correction (dividing the significance criterion by the number of tests; see Section 1 of the supplementary material for a derivation) in order to control the probability of finding at least one significant outcome when all the null hypotheses are true. However, as Westermann and Hager (1986) noted, the Bonferroni correction is not needed (and is actually conservative) if scientists conclude support for an effect only when all tests produce significant outcomes. Here, we further argue that in some situations, scientists should apply a reverse Bonferroni correction in order to maintain an intended Type I error rate.

This paper is organized as follows. We first explain how reverse Bonferroni applies to direct replications. This situation is mathematically straightforward, but is also where metaanalytical alternatives exist. We then turn to more complicated situations involving conceptual replications and multiple tests within an experiment. To help identify the requirements and limitations of the reverse Bonferroni method, we then describe some situations where the method should not be used. We 
finish with a brief comparison of reverse Bonferroni to some meta-analytic methods.

\section{Reverse Bonferroni correction for direct replications}

Consider a scenario where scientists insist that a finding be successfully replicated before they accept the conclusions of an investigation. To convince peers about the validity of a conclusion, a scientist might plan to run three independent experiments and only conclude that an effect exists if all three experiments produce significant results. If the null hypothesis happens to be true, the probability of all three experiments producing significant outcomes when using the traditional significance criterion of $\alpha=0.05$ is $\alpha^{3}=0.000125$. That is, the compound Type I error rate for the set is much smaller than that for the individual experiments.

It might seem like there is no harm in being extra conservative, but a very low compound Type I error rate dramatically reduces compound power, that is, the probability that each result is statistically significant given a true non-zero effect. For example, a two-sample, two-tailed $t$-test with a standardized effect size of $d=0.5, \alpha=0.05$, and $n_{1}=n_{2}=64$ has a power of 0.8 , while the compound probability that three such independent experiments testing the same effect produce significant effects is only $0.8^{3}=0.512$. By requiring all three experiments to be successful, the scientist thus has a rather low chance of concluding support for the effect even if it exists. To increase the compound power to 0.8 by increasing the sample size for each experiment, the scientist would need 95 participants in each group. In this case, each individual experiment has a power of 0.929 and the compound power is thus $0.929^{3}=0.8$.

Power can also be increased by relaxing the criterion for each test, that is, by adjusting it in the opposite direction as compared to standard Bonferroni correction. Using reverse Bonferroni, this can be done in such a way that the compound Type I error rate does not exceed 0.05 . When using reverse Bonferroni, a compound Type I error rate, $\alpha$, is satisfied by defining a modified significance criterion $\alpha_{r}=\sqrt[k]{\alpha}$ for each of the $k$ tests. This correction for the set of three experiments mentioned above with $\alpha=0.05$ gives $\alpha_{r}=\sqrt[3]{0.05} \approx 0.368$. This might seem like a very high number, and it indeed would be for a single test. However, the compound error rate, i.e. the likelihood of all three experiments producing Type I errors, is only $0.368^{3}=0.05$. With $d=0.5$ and $n_{1}=n_{2}=64$, like in the above example, and with this increased criterion, each experiment now has a power of 0.973 and the compound power for all three experiments producing significant effects is $0.973^{3}=$ 0.921. By using reverse Bonferroni, we have thus dramatically increased the compound power (from 0.512 to 0.921 ) simply by relaxing the criterion to maintain an appropriate Type I error rate. We can also use reverse Bonferroni to plan an appropriate sample size. Using the corrected $\alpha_{r}=0.368$, we achieve a power of 0.929 for each experiment and thus a compound power of $0.929^{3}=0.8$ already with a sample of $n_{1}=n_{2}=45$. This should be compared to the non-corrected example in the previous paragraph, where 95 participants were needed in each group to get the same power. In summary, the reverse Bonferroni correction maintains power with fewer than half as many participants as for the uncorrected tests, while restricting the compound Type I error rate to the desired 0.05 rate.

In which situations is the reverse Bonferroni correction applicable? When using traditional Bonferroni correction, scientists must identify a "family" of tests that are relevant to a common theory, idea, or mechanism (Rubin, 2017). The same requirement applies to the use of the reverse Bonferroni correction. Further, reverse Bonferroni requires that the null hypotheses are either all true or all false. In this setting, the tests differ in their significance status only due to random sampling or to differences in experimental power. One situation that has this property is when experiments are direct replications, and we envision reverse Bonferroni playing an important role in interpreting planned replication attempts. Currently, metaanalytical methods are used to pool data across direct replications and, as we show in the supplementary material (section 2, especially Figure S1), they are superior to reverse Bonferroni if the significance status of individual tests is not considered important. However, scientists do seem to care about the outcome of individual experiments as is evident by how scientific arguments depend on successful outcomes across multiple studies (Open Science Collaboration, 2015; Prinz, Schlange, \& Asadullah, 2011; but see Francis, 2014). Moreover, as we show in the following sections, reverse Bonferroni can be applied in some situations where metaanalytic methods are not appropriate.

\section{Using reverse Bonferroni for conceptual replications}

Sometimes experiments with very different measurements support the existence of an effect by providing converging evidence for a conclusion. To understand how reverse Bonferroni behaves in such a situation, consider a set of four experiments with different designs (conceptual replications). We assume that the scientist running these tests can argue that they investigate the same underlying phenomenon, so if the null is true for one experiment it is also true for the other experiments.

- Experiment 1 is a two-sample, one-tailed (positive) $t$-test with $n_{1}=n_{2}=50$ that compares independent means. The 
test compares the null hypothesis $H_{0}: \mu_{1}-\mu_{2}=0$ to the alternative hypothesis $H_{a}: \mu_{1}-\mu_{2}>0$.

- Experiment 2 tests for a non-zero correlation with $n=50$. The test compares the hypotheses: $H_{0}: \rho=0$ and $H_{a}: \rho>$ 0 .

- Experiment 3 is a one-sample $t$-test with $n=50$. The hypotheses are: $H_{0}: \mu=0$ and $H_{a}: \mu>0$.

- Experiment 4 is a one-sample test (z-test) of proportions with $n=50$. The hypotheses are: $H_{0}: P=0.5$ and $H_{a}: P>$ 0.5 .

We consider two different effect sizes: 0 and 0.3 . When the effect size is 0 , the null hypothesis is true for all experiments. When the effect size is 0.3 , every alternative hypothesis is true but in different quantitative ways. For Experiment 1, the effect refers to the standardized effect for the difference of means (Cohen's $d$ ). For Experiment 2, the effect refers to the population correlation. For Experiment 3, the effect refers to the standardized population mean. For Experiment 4, the effect refers to the increase of the population proportion from 0.5. Although related to a common conclusion, these experiments do not necessarily have to measure the same effect. Indeed, the common value of 0.3 actually refers to very different population effect sizes for the different tests. Thus, it would be inappropriate to pool the effect sizes using meta-analysis methods (fixed-effect or random effect). However, as discussed in section 3 of the supplemental material, it is possible to apply Fisher's meta-method of pooling $p$-values to this situation (see Figure S2 in the supplementary material).

Since there are four independent experiments, the reverse Bonferroni method uses a significance criterion of $\alpha_{r}=\sqrt[4]{0.05}$ $\approx 0.47$ for each experiment, with the stipulation that the scientist concludes that an effect exists only if each of the four experiments produces a significant outcome. When the true effect size is 0 (the null hypothesis for each test is true), the compound Type I error rate when using the adjusted criterion is $0.47^{4}=0.05$. When the effect size is 0.3 , the compound power is $0.923 \times$ $0.980 \times 0.980 \times 0.999=0.89$. In comparison, if the traditional $\alpha=0.05$ is used for each test, the compound Type I error rate is $0.05^{4}=0.00000625$ and the compound power is $0.438 \times 0.683 \times$ $0.673 \times 0.999=0.2$. Thus, the reverse Bonferroni correction dramatically increases the compound power while keeping the compound Type I error rate at 0.05 .

\section{Using reverse Bonferroni for multiple tests within an experiment}

The reverse Bonferroni logic also applies when a conclusion requires multiple significant outcomes within a single experiment, provided that the null hypotheses are jointly either all true or all false. Notably, commonly used meta-analytic methods (fixed, random, or Fisher's) ignore dependencies between measurements. This shortcoming could be addressed with methods that model the covariance between outcomes (Hedges, Tipton, \& Johnson, 2010), use multilevel models (Fernández-Castilla et al., 2020), or use multivariate metaanalytic methods (Jackson, Riley, \& White, 2010). However, even these approaches are inappropriate for pooling results within a single experiment if the test results measure fundamentally different effects, because the effects should in this case not be pooled together at all. We present three examples, based on published studies, where the reverse Bonferroni method can be applied to multiple tests within a single experiment.

\section{Multiple tests of memory performance}

A scientist comparing two methods for improving memory might analyze a within-subjects data set in two ways (e.g., Nairne, Thompson, \& Pandeirada, 2007). First, she might compute a difference score between the mnemonic methods for each participant and use a one-tailed $t$-test to examine if the mean difference is greater than zero. Second, she might test whether a majority of participants score higher with method 1 than with method 2. Importantly, if there is truly no difference between the methods and differences between participants are normally distributed from a fixed population (typical assumptions for a $t$-test), the null hypothesis is true for both tests. Suppose that the scientist will conclude better memory for method 1 only when both of the following tests are significant:

- A one-tailed dependent $t$-test that compares the mean number of words recalled for each method. The test compares the hypotheses: $H_{0}: \mu_{1}-\mu_{2}=0$ and $H_{a}: \mu_{1}-\mu_{2}>$ 0 .

- A one-tailed one-sample test ( $z$-test) of proportions to investigate whether a majority of subjects do better with method 1 than with method 2 . The test compares the hypotheses: $H_{0}: P=0.5$ and $H_{a}: P>0.5$.

Given the dependencies between the statistics, it is not obvious how to analytically derive the compound Type I error rate. We estimated it by generating 50,000 simulated experiments (see the file MeansProportions/RBMeansAndProportions.R at the Open Science Framework (OSF)). Each simulated experiment drew $n=30$ pairs of random samples from a multidimensional normal distribution with $\mu_{1}=\mu_{2}=0, \sigma=1$, and correlation $\rho=0.5$ (the data in Study 2 of Nairne et al. (2007) have a correlation of $r=0.58$ ). Since both null hypotheses are true for this sampling procedure and we used $\alpha=0.05$ for each test, the simulated Type I error rate for each test is very close to 0.05 (minor deviations from 0.05 are due to random sampling). To conclude that there is a difference between the memory methods both tests are required to be significant, and the simulations (see 
the file MeansProportions/FindAlphaRB.R at the OSF) indicate that this happens only at a rate of 0.025 (if the two tests were independent, this rate would be 0.0025 ). In order to find an appropriate reverse Bonferroni criterion, we created a simple staircase algorithm that found a significance criterion, $\alpha_{r}=$ 0.08 , that produces a compound Type I error rate just below 0.05 . Changes to the value of the population means, standard deviation, and correlation did not impact the Type I error rate in our simulations, which readers can verify using the code at the OSF.

If there actually is a difference between the two memory methods (say, $d=0.2$ ) and the correlation across participants is $\rho=0.5$, a sample size of $n=337$ is needed to achieve $90 \%$ power for finding significance in both tests using the traditional $\alpha=0.05$. In contrast, using the reverse Bonferroni criterion of $\alpha_{r}=0.08$ gives the same compound power with only $n=$ 279 participants.

\section{Tests of reaction time and accuracy}

In some behavioral experiments, scientists measure both the time taken to respond to a stimulus and the accuracy of the response. While these two performance measures sometimes trade off against each other (e.g., faster performance corresponds to lower accuracy), there are situations where they both improve. For example, in monkeys both reaction time and accuracy for identifying the overall direction of a cloud of moving dots improved as dot motion coherency increased (Palmer, Huk, \& Shadlen, 2005). Suppose a scientist wonders if variation in the strength of an illusory contour (Francis \& Wede, 2010) has the same kind of effect. To support the conclusion, she would require statistical significance for two related tests that compare performance in strong and weak illusory contour conditions:

- A one-tailed independent $t$-test that compares the mean reaction time for the strong and weak conditions. The test compares the hypotheses: $H_{0}: \mu_{\text {Strong }}-\mu_{\text {Weak }}=0$ and $H_{a}: \mu_{\text {Strong }}-\mu_{\text {Weak }}<0$.

- A one-tailed two-sample test (z-test) that compares the proportion correct for the strong and weak conditions. The test compares the hypotheses: $H_{0}: P_{\text {Strong }}-P_{\text {Weak }}=$ 0 and $H_{a}: P_{\text {Strong }}-P_{\text {Weak }}>0$.

We estimated the compound error rate of these two tests by generating 50,000 simulated experiments using a diffusion model of decision making (Singmann et al., 2018). Each simulated experiment drew $n=150$ samples for each condition using the same data generation parameters (the code is available at the OSF in the file ReactionTimeAccuracy/SpeedAccuracy.R). Since both null hypotheses are true for this sampling procedure, when using $\alpha=0.05$ the simulated Type I error rate for each test is very close to 0.05 . However, a conclusion to indicate a difference between the conditions requires both tests to be significant, and that happens only at a rate of 0.0026 (this value is very close to the 0.0025 compound rate that would be expected for two independent tests, but independence cannot generally be assumed for the diffusion model). Our staircase algorithm (ReactionTimeAccuracy/FindAlphaRB.R) indicates that using reverse Bonferroni with a criterion of $\alpha_{r}=0.22$ produces a compound Type I error rate of 0.05 .

To investigate power, we generated data for the strong condition from a diffusion model having a starting point parameter of $z=0.325$, while for the data of the weak condition we used a parameter of $z=0.25$. This parameter difference tends to make the reaction times shorter (by around $10 \mathrm{~ms}$ ) and accuracy better (an increase in proportion correct of around 0.13 ) for the strong condition compared to the weak condition. However, despite these real effects, with $n=150$ and using the traditional $\alpha=0.05$ for each test, the probability of both tests producing a significant result (power) is only 0.35 . In contrast, using the reverse Bonferroni criterion of $\alpha_{r}=0.22$, the probability of both tests producing significant results is 0.73 .

\section{Multiple tests of sets and subsets}

Before concluding support for an effect, scientists sometimes require a significant result across both a full group and a subgroup of the data. For example, to investigate the facial feedback hypothesis (forming a particular facial expression subconsciously influences emotion), Marsh, Rhoads, and Ryan (2018) ran two tests on their data set. The first one was a within-subjects test that compared emotion ratings across two facial conditions. A second between-subjects test compared emotion ratings across facial conditions for the first trial only; this second test was similar to the between-subjects design of a previous study (Strack, Martin, \& Strepper, 1988). To conclude a difference between the two conditions, the scientists required significance for both tests. If the two tests were independent, the adjusted criterion would be $\alpha_{r}=\sqrt{0.05} \approx 0.22$. However, since one test is based on a subset of the data in the other test, the two tests are dependent, and the adjusted criterion will be lower. The reverse Bonferroni method gives an adjusted significance criterion of $\alpha_{r}=0.104$ for each test (for details see supplementary material, section 4). Consistent with previous examples, the choice of the criterion has a big impact on the power of the tests. If $d=0.5$, and decisions are made using the traditional $\alpha=0.05, n=100$ gives a probability of both tests producing a significant result (compound power) of 0.79. In contrast, when using the reverse Bonferroni criterion of $\alpha_{r}=0.104$, the compound power is 0.89 .

It is also possible to apply reverse Bonferroni to conclusions based on multiple outcomes within and between 
experiments. The logic is much the same as above, and an example is provided in Section 5 supplemental material.

\section{When reverse Bonferroni should not be used}

For the logic of the reverse Bonferroni method to be valid, the null hypotheses of the different tests have to be either all true or all false. Failing to meet this strict requirement may have severe statistical consequences. In an extreme case, one might imagine the researcher "helping" the significance of a test result by adding unrelated and trivial tests that the researcher knows are bound to give significant results to the group of interest. Adding trivial (i.e., surely statistically significant) tests, will increase alpha for every test, thereby artificially increasing compound power. This problematic situation can be contrasted against standard Bonferroni correction where including non-relevant tests only makes each test stricter, which is not desirable but does not compromise the interpretation of a statistically significant outcome. In order to convince readers that the reverse Bonferroni correction is applicable in a certain situation, the researcher will have to argue that the null hypothesis of every test should be expected to be uniformly false or uniformly true. The strongest argument will probably be made in a pre-registration document that describes the set of tests, how they will be analyzed, and why reverse Bonferroni applies. The shared status of the null hypothesis was a key characteristic of the examples using the reverse Bonferroni method in the previous sections. However, there are many situations where multiple null hypotheses might be relevant to a conclusion but not all true or all false. To better understand this requirement, it may be useful to consider an example where reverse Bonferroni should not be used.

Suppose a scientist has developed a new mnemonic method to improve memory for a list of words. The scientist hopes that the new method is better than existing methods, so he identifies the four best mnemonic methods in the current literature. He runs a new experiment where participants are assigned to use one of the methods (Nairne, Pandeirada, \& Thompson, 2008). To conclude that the new method is the best one, the scientist requires it to be significantly better than every alternative method. Thus, there are four tests of the form:

- A one-tailed independent $t$-test compares the mean number of words recalled for the new method (method 1) and an alternative method $j(\operatorname{method} 2,3,4$, or 5$)$. The test compares the hypotheses: $H_{0}: \mu_{1}-\mu_{j}=0$ and $H_{a}: \mu_{1}-\mu_{j}>0$.

At first glance, this might seem like a good situation for using reverse Bonferroni. Indeed, when all the null hypotheses are true, simulated experiments reveal that the compound
Type I error rate for concluding that the new method is the best is only 0.0027 . Likewise, if the new method truly is the best, the power of the procedure might be quite low because the conclusion requires multiple significant outcomes. If the four tests were independent, the reverse Bonferroni adjusted criterion would be $\alpha_{r}=\sqrt[4]{0.05} \approx 0.47$. However, since all four tests include the data for method 1 , the tests are dependent and the adjusted criterion will therefore be lower. Our search algorithm finds a reverse Bonferroni criterion of $\alpha_{r}=0.251$ (see the file ImproperUsesOfRB/BestMemoryMethod/ FindAlphaRB.R at the OSF). If all the null hypotheses are true, using this adjusted criterion for each test will result in a compound Type I error rate of approximately 0.05 .

However, reverse Bonferroni should not be applied here because there is no reason to believe that when method 1 is equivalent to method 2 , it must also be equivalent to the other methods. To see why this is a problem, suppose that method 1 really is better than methods $3-5$ and the sample sizes are large enough for each of those comparisons to almost always produce a significant outcome (power is approximately 1 ). Further, suppose that method 1 is not actually better than method $2\left(H_{0}: \mu_{1}-\mu_{2}=0\right.$ is true); it would thus be a Type I error to conclude that the new method is better than all the other methods. In this case, the compound Type I error rate will be equal to the criterion used for the comparison between the two equivalent methods (methods 1 and 2). If the scientist used the reverse Bonferroni significance criterion of $\alpha_{r}=$ 0.251 for each test, the compound Type I error rate would also be 0.251 . Considering this worst-case scenario for compound Type I error control, the scientist should stick to $\alpha=0.05$ for each test.

\section{Comparing reverse Bonferroni to meta-analytic methods}

It is common for scientists to require multiple significant outcomes when making decisions about the existence of an effect or concluding support for a theory. Current practice seems to largely ignore the impact that multiple tests have on the Type I error rate and power for the decisions. The reverse Bonferroni method is, to our knowledge, the first method to address the deleterious impact that requiring multiple tests imposes on compound error rates.

Whereas reverse Bonferroni adjusts significance to control the compound Type I error rate, meta-analytic methods ignore the statistical significance of individual tests and instead pool effect sizes across experiments. Which approach is better depends on whether scientists care more about estimating effect sizes or about a pattern of statistical significance (e.g., replication). Even though they emphasize different approaches to science, the methods are not in direct conflict. Scientists could 
use reverse Bonferroni to evaluate significance for individual experiments and still perform a meta-analysis to estimate an effect size. Importantly, effect size estimation only makes sense if there is a common population effect (that perhaps differs by a linear transformation, or is part of a distribution of effect sizes - as in a random-effects meta-analysis), but reverse Bonferroni can be used in situations where widely differing population effects are involved. Section 2 of the supplemental material describes simulations that compare reverse Bonferroni and a fixed effect meta-analysis for a situation where both methods apply.

Fisher's combined probability test (Fisher's method) can be used to investigate whether multiple independent tests all have a true null hypothesis or at least one of the tests has a true alternative hypothesis. This method is similar to meta-analysis in that it does not consider the statistical significance of individual experiments, but it is similar to reverse Bonferroni in that it can consider widely differing population effects. The calculations of this method are based on the reported $p$-values and are described in section 3 of the supplemental material. The supplemental material also demonstrates that Fisher's method is more powerful than reverse Bonferroni for situations where both methods apply. A limitation of Fisher's method is that (except for special cases, e.g., Brown, 1975) it requires independent tests, which is not necessary for application of reverse Bonferroni.

When they are applicable, we believe that meta-analytic approaches are better than basing decisions on multiple significant outcomes. However, it is clear that some scientists feel otherwise (see Francis, 2014), and we recognize that there are situations (described in previous sections), where multiple tests cannot be avoided. In these cases, we recommend the reverse Bonferroni method, if it applies.

\section{Conclusions}

We have introduced the reverse Bonferroni correction for hypothesis testing where multiple significant test outcomes are required and described cases where it could be used. In every case, the reverse Bonferroni correction dramatically increases power and appropriately sets the compound Type I error rate.

Is it reasonable to require multiple successful outcomes in order to claim support for a hypothesis? Common intuition in this type of situation is often wrong. For example, when conducting three independent studies, each with a power of 0.8 , the likelihood of achieving significant results in all three studies is only $0.8^{3} \approx 0.5$. Thus, we only have about a $50 \%$ chance of getting consistent support for the effect. We are not necessarily advocating that scientists base their conclusions on the outcomes of multiple tests, especially if a meta-analytical pooling of effects is appropriate (Hedges \& Olkin, 1985) or posterior probabilities can be estimated using Bayesian methods. But whether basing conclusions on one test or multiple tests, scientists should design fair hypothesis tests. Whenever possible, the conclusions from a statistical analysis based on hypothesis tests should have a controlled compound Type I error rate if the null hypotheses are true and high compound power if the null hypotheses are false. Currently, conclusions relying on multiple successful uncorrected tests are overly conservative, with a low compound Type I error rate and accordingly low compound power (Francis, 2012; Schimmack, 2012). In contrast to the ad hoc approaches that are currently in practice, the reverse Bonferroni correction provides a principled way to promote good experimental designs that provide answers to complex scientific questions.

There is an obvious danger of the reverse Bonferroni method enabling yet another questionable research practice that could be exploited by scientists who seek ways to support their conclusions (Simmons et al., 2011). Indeed, if it is used inappropriately in combination with other questionable research practices, the compound Type I error rate might even approach 1. In particular, a scientist whose study has failed to produce significance with the traditional $\alpha=0.05$ criterion (e.g., the study produces $p=0.2$ ) might run a replication study and then claim significance for both studies using a reverse Bonferroni criterion of $\alpha_{r}=\sqrt{0.05} \approx 0.22$. This approach would be invalid because the decision on whether to use the correction must not depend on the outcome of the first test. In hypothesis testing, the decision-making procedure should always be specified in advance. Considering that the reverse Bonferroni correction can induce large biases when implemented inappropriately, we recommend it be used only for pre-registered investigations (Munafò et al., 2017). Indeed, being able to use reverse Bonferroni to relax the significance criterion provides strong encouragement for scientists to plan their analysis procedures prior to data collection.

Supplementary Information The online version contains supplementary material available at https://doi.org/10.3758/s13423-020-01855-z.

Author note The authors declare no competing interests. R code for all simulations is available at the Open Science Framework https://osf.io/ gsjr7/

\section{References}

Brown, M. (1975). A method for combining non-independent, one-sided tests of significance. Biometrics, 31(4), 987-992.

Cohen, J. (1994). The Earth is round $(\mathrm{p}<.05)$. American Psychologist, 49(12), 997-1003.

Fernández-Castilla, B., Jamshidi, L., Declercq, L., Beretvas, S. N., Onghena, P. \& Van den Noortgate, W. (2020). The application of meta-analytic (multi-level) models with multiple random effects: A systematic review. Behavior Research Methods, https://doi.org/10. 3758/s13428-020-01373-9 
Francis, G. (2012). Too good to be true: Publication bias in two prominent studies from experimental psychology. Psychonomic Bulletin \& Review, 19(2), 151-156.

Francis, G. (2014). The frequency of excess success for articles in Psychological Science. Psychonomic Bulletin \& Review, 21, 11801187.

Francis, G., \& Wede, J. (2010). Properties of long-range illusory contours produced by offset-arcs. Perception, 39(11), 1466-1475. https://doi. org $/ 10.1068 / \mathrm{p} 6613$

Hedges, L. V, \& Olkin, I. (1985). Statistical methods for meta-analysis. San Diego: Academic Press.

Hedges, L. V., Tipton, E., \& Johnson, M. C. (2010). Robust variance estimation in meta-regression with dependent effect size estimates. Research Synthesis Methods, 1, 39-65.

Jackson, D., Riley, R., \& White, I. R. (2010). Multivariate meta-analysis: Potential and promise. Statistics in Medicine, 30, 2481-2498.

John, L. K., Loewenstein, G., \& Prelec, D. (2012). Measuring the prevalence of questionable research practices with incentives for truth telling. Psychological Science, 23(5), 524-532. https://doi.org/10. 1177/0956797611430953

Marsh, A. A., Rhoads, S. A., \& Ryan, R. M. (2018). A multi-semester classroom demonstration yields evidence in support of the facial feedback effect. Emotion. https://doi.org/10.1037/emo0000532

Munafò, M. R., Nosek, B. A., Bishop, D. V. M., Button, K. S., Chambers, C. D., Percie Du Sert, N., ... Ioannidis, J. P. A. (2017). A manifesto for reproducible science. Nature Human Behaviour, 1(1), 1-9. https://doi.org/10.1038/s41562-016-0021

Nairne, J. S., Pandeirada, J. N. S., \& Thompson, S. R. (2008). Adaptive memory: The comparative value of survival processing. Psychological Science, 19(2), 176-180.

Nairne, J. S., Thompson, S. R., \& Pandeirada, J. N. S. (2007). Adaptive memory: Survival processing enhances retention. Journal of Experimental Psychology: Learning Memory and Cognition, 33(2), 263-273. https://doi.org/10.1037/0278-7393.33.2.263

Nosek, B. A., Spies, J. R., \& Motyl, M. (2012). Scientific utopia: II. Restructuring incentives and practices to promote truth over publishability. Perspectives on Psychological Science, 7(6), 615631. https://doi.org/10.1177/1745691612459058
Open Science Collaboration. (2015). Estimating the reproducibility of psychological science. Science, 349(6251). https://doi.org/10.1126/ science.aac4716

Palmer, J., Huk, A. C., \& Shadlen, M. N. (2005). The effect of stimulus strength on the speed and accuracy of a perceptual decision. Journal of Vision, 5, 376-404. https://doi.org/10.1167/5.5.1

Prinz, F., Schlange, T., \& Asadullah, K. (2011). Believe it or not: How much can we rely on published data on potential drug targets? Nature Reviews Drug Discovery, 10(9), 712-712. https://doi.org/ 10.1038/nrd3439-c1

Roediger, H. L. (2012). Psychology's woes and a partial cure: The value of replication. APS Observer, 25(2), 27-29.

Rubin, M. (2017). Do $p$ values lose their meaning in exploratory analyses? It depends on how you define the familywise error rate. Review of General Psychology, 21(3), 269-275.

Schimmack, U. (2012). The ironic effect of significant results on the credibility of multiple-study articles. Psychological Methods, 17(4), 551-566. https://doi.org/10.1037/a0029487

Simmons, J. P., Nelson, L. D., \& Simonsohn, U. (2011). False-positive psychology: Undisclosed flexibility in data collection and analysis allows presenting anything as significant. Psychological Science, 22(11), 1359-1366. https://doi.org/10.1177/0956797611417632

Singmann, H., Brown, S., Gretton, M., Heathcote, A., Voss, A., Voss, J., \& Terry, A. (2018). Package "rtdists." https://doi.org/10.1162/neco. 2008.12-06-420

Strack, F., Martin, L. L., \& Strepper, S. (1988). Inhibiting and facilitating conditions of facial expressions: A non-obstrusive test of the facial feedback hypothesis. Journal of Personality and Social Psychology1, 54(5), 768-777.

Westermann, R., \& Hager, W. (1986). Error probabilities in educational and psychological research. Journal of Educational Statistics, 11(2), 117-146.

Publisher's note Springer Nature remains neutral with regard to jurisdictional claims in published maps and institutional affiliations. 